\title{
Etiological Definition and Diagnostic Work-Up
}

\author{
Marco Merlo, Marco Gobbo, Jessica Artico, Elena Abate, \\ and Stefania Franco
}

\section{Abbreviations and Acronyms}
AL Amyloid light chain
ATTR Amyloid transthyretin
BNP Brain natriuretic peptide
CMR Cardiovascular magnetic resonance
CS Cardiac sarcoidosis
DCM Dilated cardiomyopathy
EF Ejection fraction
EMB Endomyocardial biopsy
FDG Fluorodeoxyglucose
HF Heart failure
HRS Heart Rhythm Society
ICD Implantable cardioverter defibrillator
LGE Late gadolinium enhancement
LV Left ventricle
LVRR Left ventricular reverse remodeling
RV Right ventricle

\footnotetext{
M. Merlo $(\triangle)$

Cardiovascular Department, Azienda Sanitaria Universitaria Integrata, Trieste, Italy

M. Gobbo · J. Artico · S. Franco

Cardiovascular Department, Azienda Sanitaria Universitaria Integrata, University of Trieste (ASUITS), Trieste, Italy
}

E. Abate

Cardiovascular Department, Azienda Sanitaria Universitaria Integrata, University of Trieste (ASUITS), Trieste, Italy

Klinik für Innere Medizin 3, Universitätsklinikum Halle (Saale), Halle (Saale), Germany e-mail: Elena.Abate@uk-halle.de 


\subsection{Clinical Presentation}

Most patients affected by dilated cardiomyopathy (DCM) present between the ages of 20 and 60, but the disease can occur also in children and older adults. They are most commonly Caucasian males. Furthermore, with respect to other heart failure etiologies, the comorbidity profile of DCM patients is very low. Finally, due to the frequent long-standing asymptomatic left ventricular dysfunction, these patients are often scarcely symptomatic for heart failure (HF) at diagnosis in spite of importantly remodeled left ventricle. Useful patterns in diagnosing DCM are:

1. Heart failure symptoms (progressive dyspnea with exertion, impaired exercise capacity, orthopnea, paroxysmal nocturnal dyspnea, and peripheral edema)

2. Incidental detection of asymptomatic cardiomegaly

3. Incidental detection of left bundle branch block (e.g., sport screening in countries with ECG sport screening)

4. Symptoms related to coexisting arrhythmia, conduction disturbance, thromboembolic complications

5. Sudden death

6. Familial screening

\subsection{Etiological Classification: A Critical Issue in Clinical Management of DCM}

DCM prognoses have changed dramatically in the last decades [1]. This aspect is due to an improvement in HF therapy, both pharmacologic and non-pharmacologic (e.g., devices and MitraClip()), promoting left ventricular reverse remodeling (LVRR). However, the diagnostic effort facing a newly discovered DCM phenotype is critical to address a tailored therapy and to improve the LVRR amount and longterm survival [2]. In fact, DCM is a generic term that encases several different diseases. Timing is also a crucial aspect since delaying an accurate etiological definition of nonischemic DCM could mean to increase the event rate. In this sense a red flags approach appears important, and advanced diagnostic tools should be used not in every patient but in whom red flags suggest utility rather than futility [3].

\subsubsection{Need of Reclassification of the Disease During Follow-Up}

The overall improvement of prognosis in DCM makes quite frequent to see in clinical practice patients with $>10$ years of disease. In these patients an eventual worsening of systolic function or arrhythmias could be explained by a progression of the disease but also by a developed superimposed coronary artery disease, valvulopathy, or active myocarditis. In this sense repeated etiological classification of disease is advocated and appears crucial periodically during follow-up [4]. 


\subsection{Exclusion of Reversible Causes of Left Ventricular Dysfunction/Dilation}

It is pivotal to exclude possible removable causes of left ventricular dysfunction [4]. First and by far most important is to exclude an ischemic cardiomyopathy that is conventionally distinguished from DCM by the presence of $>50 \%$ stenosis in the left main stem, proximal left anterior descending artery, or two or more epicardial coronary arteries on invasive or computed tomography coronary angiography (2). Late gadolinium enhancement (LGE) at cardiovascular magnetic resonance (CMR) provides an alternative approach and may identify prior myocardial infarction (subendocardial or transmural LGE) in as many as $13 \%$ of patients with suspected DCM and unobstructed coronary arteries [5]. In addition to ischemic cardiomyopathy, DCM must also be distinguished from other nonischemic cardiomyopathies and physiological adaptations that may generate similar patterns of left ventricle (LV) remodeling [6]. One example is represented by valvular heart disease associated with left ventricular systolic dysfunction.

Hypertensive dilated cardiomyopathy is a challenging entity from diagnostic standpoint. These patients usually are older and with more comorbidities, tolerating higher doses of drugs, or with high pressure in spite of LV dysfunction and a septal thickness of more than $12 \mathrm{~mm}$ [7]. However, it is still unknown why only few hypertensive patients develop LV systolic dysfunction in the absence of concomitant coronary artery disease. A genetic background favorable to develop DCM is likely, but future focused studies are advocated to elucidate this issue.

The term "idiopathic DCM" is often used in clinical practice and in some series accounts for $20-30 \%$ of nonischemic HF. However, the approach to a patient with nonischemic DCM rarely seeks reversible factors other than hypertension, valve disease, and congenital heart disease. Other examples of commonly overlooked or underappreciated reversible environmental triggers for LV dysfunction include sustained supraventricular arrhythmias or very frequent ventricular ectopic beats, which can lead to tachycardia-induced cardiomyopathy; substance abuse (e.g., alcohol, cocaine); acute emotional stress or chemotherapies that cause catecholamine (i.e., Takotsubo) or toxin-induced cardiomyopathies; and systemic autoimmune disorders (e.g., Churg-Strauss syndrome and sarcoidosis). New-onset HF with LV dysfunction occurring during pregnancy or the postpartum period could identify a peripartum cardiomyopathy. Confirmation of active myocarditis as the cause of recent onset severe HF is particularly important as it may require investigations (e.g., endomyocardial biopsy) [8].

Accordingly, a comprehensive integrated approach, including third-level diagnostic tools, should be systematically implemented in clinical practice to remove every possible reversible cause through specific therapeutic interventions. This issue appears essential to promote left ventricular reverse remodeling and subsequent outcome improvement [2] (Fig. 4.1). 


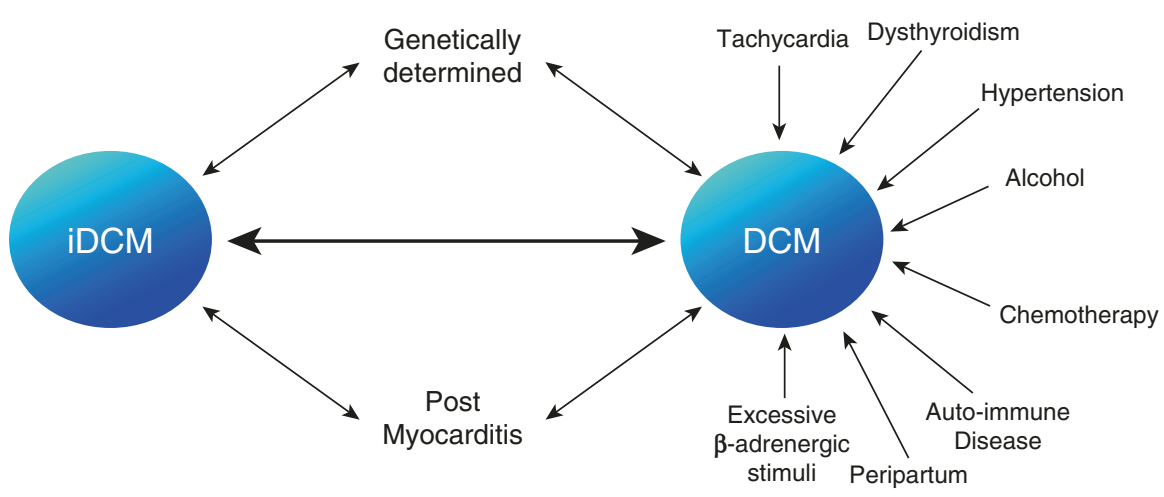

Fig. 4.1 Etiological characterization of DCM. DCM dilated cardiomyopathy, IDCM idiopathic dilated cardiomyopathy. From Merlo et al. Evolving Concepts in Dilated Cardiomyopathy, EJHF.2018. 20(2):228-239

\subsection{Diagnostic Work-Up in New-Onset LV Dysfunction/ Dilation: A "Red Flags" Approach}

As previously stated, DCM may present with multiple clinical scenarios. However, the clinical approach to a suspected DCM requires a step-by-step work-up. It is crucial to start from the family and personal history, to perform a comprehensive physical examination and to interpret all the available diagnostic tools. Rapezzi and colleagues first described the so-called red flags approach in cardiomyopathies to guide the selection of the appropriate diagnostic techniques [3]. The easily missed boxes (see below) provide some important examples of this approach and of difficulties of differential diagnosis in approaching a newly diagnosed nonischemic DCM.

\subsubsection{Personal and Family History}

In the adulthood, the onset of the disease is generally observed during the third or fourth decade of life. This is "unusual" in classic genetic diseases. Since genetic forms account for $20-50 \%$ of DCM cases, the first clinical examination should include a very careful assessment of the patient's family history [9]. The recording of a complete family pedigree is helpful in determining the possible mode of genetic transmission (autosomal dominant, autosomal recessive, X-linked, matrilinear) and in detecting other cardiac and non-cardiac traits associated with DCM. The pedigree is by far the most important genetic tool in the systematic approach to DCM [9]. Importantly, a negative family history does not rule out a genetic form of DCM as de novo genetic mutations can be responsible for sporadic DCM. Systematic familial screening is an important way of diagnosis shedding light on early phases of disease with favorable prognosis [10]. 
A distinct clinical entity is the DCM formed in pediatric patients. The prognosis in this specific population is poorer than in adulthood, carrying more HF and arrhythmic events. This translates in a need of more aggressive treatments and follow-up in pediatric DCMs [11], implicating difficult clinical choices due to the age of the patients. The reasons of this particularly aggressive form in children are largely unknown. Other forms of LV dysfunction, such as active myocarditis, appear to carry an ominous prognosis in children [12]. It is possible that specific immunologic pathways could be involved, but this represents an important field for future research.

\subsubsection{Symptoms and Physical Examination}

Clinically, signs and symptoms of heart failure often characterize the onset of the disease, but young individuals can remain asymptomatic for a long time despite having LV dysfunction. A history of palpitations and syncope should be carefully investigated, as they can be the clinical expressions of serious ventricular arrhythmias. Neurologic examination is of paramount importance. A search for multisystem involvement should be part of the clinical examination, in particular looking for signs of skeletal myopathy or neurosensory disorders [3]. For example, cases of DCM associated with learning difficulties, blindness, and deafness should be recognized.

\subsubsection{Lead Electrocardiogram}

Historically, electrocardiogram in DCM has been considered non-specific. However, in some cases it can provide clues to specific forms of DCM. For example, posterolateral pseudonecrosis (that requires exclusion of true necrosis with coronary angiogram) is typical of dystrophin-related DCM. Atrioventricular blocks (of various degrees) can suggest a mutation in LMNA and are usually related to an important arrhythmic burden [13]. Sinus bradycardia and atrial standstill have been associated to myotonic dystrophy and Emery-Dreifuss muscular dystrophy [3]. Other important red flags include low QRS voltages, right bundle branch conduction abnormalities, and anterolateral/ inferior negative $\mathrm{T}$ waves which can lead to a diagnosis of biventricular or left-dominant arrhythmogenic cardiomyopathy [14]. Left bundle branch block and left atrial enlargement are usually markers of long-standing disease, the former also having prognostic and therapeutic implications (i.e., resynchronization therapy) [15]. Finally, in contrast to other cardiomyopathies, there is a lack of studies on a systematic evaluation of ECG in a large cohort of DCM. These studies could provide in the future possible diagnostic and prognostic tools derived by a simple and often neglected tool as ECG.

\subsubsection{Laboratory Tests}

In $\mathrm{HF}$ with reduced ejection fraction, natriuretic peptides have clinical utility for the diagnosis and prognostic stratification. In fact, guidelines recommend dosage of 
brain natriuretic peptide (BNP) or N-terminal pro-BNP at the time of the first evaluation and systematically during follow-up [16]. However, BNP rises irrespective of HF etiology. Instead, elevations in serum creatine phosphokinase can suggest specific genetic disorders such as dystrophinopathy, laminopathy, or desminopathy [3]. Whether clinical evidence of neuromuscular involvement is found or not, in these patients a complete neurological work-up is generally warranted. Other important laboratory markers suggesting specific etiologies are high transferrin saturation and hyperferritinemia in hemochromatosis, or lactic acidosis and leucopoenia in rare forms of mitochondrial diseases [3].

\subsubsection{Genetic Testing}

While there is a general appreciation that DCM can be caused by many different disease processes, in everyday clinical practice it is often considered under the catch-all heading of "nonischemic HF" with reduced ejection fraction. However, the concept that DCM represents a family of diseases characterized by complex interactions between environment and genetic predispositions is gaining prominence as the clinical impact of a precise diagnosis is better appreciated [17]. Nowadays, after the exclusion of possible removable causes of LV dysfunction, in both familial and sporadic cases, particularly in the presence of "red flags" suggesting possible genetic forms of DCM, a genetic testing with a next-generation sequencing approach is indicated. Despite current guidelines recognize a genotype-phenotype correlation only in LMNA carriers, there is a growing amount of data that supports genotype-phenotype correlations also for other genes (i.e., desmosomal, FLNC [18], TTN [19], sarcomere) (see Chap. 5). In this sense, it is emerging a new, widely unexplored, and important overlap syndrome between DCM and right ventricular cardiomyopathy called arrhythmogenic cardiomyopathy, often determined by mutations of LMNA, FLNC, or desmosomal genes. Therefore, it exists the next future perspective of an extensive use of genetic testing in DCM, even if the current knowledge on genotype-phenotype correlation and application of precision medicine in DCM is still embryonic [20].

\subsubsection{Echocardiography}

Two-dimensional Doppler echocardiography has an important role in the diagnostic and prognostic assessment of DCM [21]. Echocardiography has the main advantage of being very practical and quite affordable making it the perfect tool for first-line diagnosis and follow-up of DCM patients. In fact, evaluation of LV ejection fraction and LV dimensions represents the first-line approach to a DCM patient and should be periodically repeated during the follow-up. Different patterns of DCM have been described according to the grade of LV dilation. Hypokinetic non-dilated cardiomyopathy (or mildly dilated cardiomyopathy) has been recently introduced as a distinct clinical entity $[6,22]$. Specific genetic forms, such as LMNA mutations, can cause isolated LV systolic dysfunction without dilatation and have a much higher arrhythmic burden [23]. A reduced LV ejection fraction with preserved LV size can 
be also observed in the early (preclinical) stages of disease and is generally associated with a good prognosis [22]. The presence of some particular features such as a restrictive LV filling pattern and non-sustained ventricular arrhythmia carries a higher risk of an adverse outcome [24, 25]. Similar clues may suggest involvement of specific genes. Alternatively, active myocarditis can also present with depressed ejection fraction but not extensive LV remodeling, frequently in association with a high arrhythmic burden $[8,12]$.

Myocardial deformation imaging techniques (e.g., speckle tracking) offer greater sensitivity than LV ejection fraction for identifying subtle abnormalities of systolic function and may assist in the early detection of disease [26].

In addition to the examination of LV systolic function and size, the presence and the severity of functional mitral regurgitation [27] have important implications for therapeutic and prognostic strategies. Left ventricular diastolic function should be systematically assessed for the estimation of left ventricular filling pressures and the identification of restrictive filling pattern [24]. The right ventricle along with the estimation of pulmonary arterial pressure is also essential in the stratification of the disease [28].

\section{Box 4.1 Easily Missed: Cardiac Amyloidosis}

Amyloidosis is a disease complex caused by extracellular deposition of insoluble abnormal fibrils composed of misfolded proteins, which can alter tissue structure and impair function of multiple organs including the heart. Types of amyloidosis which commonly affect the heart include primary systemic amyloidosis (amyloid light chain (AL)) and transthyretin amyloidosis (amyloid transthyretin), the latter of which may be acquired in older individuals (wild type) or inherited in younger patients (hereditary).

Cardiac amyloidosis usually starts as restrictive cardiomyopathy with normal or mildly depressed LV systolic dysfunction and significant diastolic HF and can progress to severe systolic dysfunction in advanced stages. Once amyloid infiltration involves the heart, prognosis significantly worsens. In fact, median survival in AL amyloidosis is $\approx 13$ months but decreases drastically to 4 months with the onset of HF symptoms [29].

Reduced QRS voltage amplitude on ECG is noted in the limb leads in $\approx 50 \%$ of cases, but the true electrocardiographic hallmark of cardiac amyloidosis is the disproportion between left ventricular wall thickness and QRS voltages [29]. A pseudoinfarct pattern in the precordial leads is another electrocardiographic feature.

In some cases, echocardiography could suggest the diagnosis and hence enhance the sensitivity of physical examination. Typical echocardiographic features of amyloidosis include thickened ventricular walls (right and left) in the setting of normal ventricular size, biatrial dilatation, the presence of a pericardial effusion, and valvular thickening without 
significant dysfunction [30]. Increased echogenicity of the myocardium, termed granular sparkling, is not very sensitive or specific when evaluated in isolation. Even if the global LV function is usually impaired only in advanced cardiac amyloidosis, longitudinal dysfunction precedes the onset of heart failure. This is best detected by strain imaging, which typically shows impairment of longitudinal strain at the base of the left ventricle, with relatively well-preserved apical strain. When strain is color coded, a "bull's eye" with an apical sparing pattern is found; it is both sensitive and specific for the diagnosis of cardiac amyloidosis [31]. In contrast CMR findings in cardiac amyloidosis are aspecific. In fact late gadolinium enhancement is simply an expression of interstitial expansion that can be frequently found in storage diseases (e.g., Anderson-Fabry and Danon disease) [30].

Scintigraphy with bone-seeking tracers (DPD Tc in Europe and PYP Tc in the USA) is an important technique in the diagnosis of amyloidosis. Myocardial uptake is strictly dependent on etiology: absent or mild in AL, present in ATTR, and variable in other rarer genetic forms [32]. A strong myocardial tracer uptake is highly sensitive for ATTR cardiac amyloidosis (both hereditary and wild type). Furthermore, specificity in relation to sarcomeric hypertrophic cardiomyopathy has also been shown to be high [30]. There are potential pitfalls since a negative scintigraphy does not rule out a diagnosis of cardiac amyloidosis and mild myocardial tracer uptake does not allow a differential diagnosis between ATTR and AL [30].

\subsubsection{Cardiovascular Magnetic Resonance}

Cardiovascular magnetic resonance (CMR) imaging is considered the gold standard for assessment of ventricular volumes and ejection fraction, enabling confident diagnosis of DCM in borderline cases and improving the characterization of disease severity in patients with known LV dysfunction.

Another crucial aspect of CMR is tissue characterization that can be useful in diagnosis of specific forms of DCM [5, 33]. The identification of myocardial edema (in T2-weighted images) suggests active myocarditis and late gadolinium enhancement (LGE) representing replacement fibrosis and is detectable in approximately one-third of cases of DCM with a distinctive mid-wall distribution, more frequently within the septal wall [5]. The presence of LGE in DCM carries important prognostic implications in terms of low probability of LV reverse remodeling and increased risk of sudden cardiac death [5, 34]. Moreover, the distribution of LGE may be suggestive of some DCM phenotypes; for instance, an inferolateral or posterolateral location is typical of muscular dystrophy, whereas subepicardial or transmural patchy distribution of LGE is suggestive of myocarditis or sarcoidosis [35]. 
Emerging CMR techniques, specifically T1 mapping, provide assessment of the interstitial fibrosis and could represent in the future a tool for early diagnosis and risk stratification of DCM [36].

CMR T2* imaging is the preferred technique for the detection and quantification of iron deposits within the myocardium in patients with hemochromatosis.

\section{Box 4.2 Easily Missed: Cardiac Sarcoidosis}

Sarcoidosis is a multisystemic inflammatory disease of unknown origin characterized by noncaseating granuloma formation in multiple organ systems. The disease affects more frequently the lung (more than $90 \%$ of patients), but it can also involve the heart, liver, spleen, skin, eyes, parotid gland, and other organs and tissues. A certain diagnosis requires histopathologic demonstration of noncaseating granulomas at lung biopsy. Clinically cardiac involvement occurs in about $5 \%$ of patients with sarcoidosis, but autopsy findings and, more recently, data based on CMR studies showed that $25-50 \%$ of patients with sarcoidosis have some degree of cardiac involvement. The principal manifestations are conduction abnormalities, ventricular arrhythmias (including sudden death), and heart failure [37, 38].

Diagnosis of cardiac sarcoidosis is challenging, and diagnostic criteria rely on the presence of noncaseating granuloma on histological examination of myocardial tissue. Among patients with extra-cardiac sarcoidosis, diagnosis of cardiac sarcoidosis is probable in the presence of reduced LVEF, unexplained ventricular tachycardia, conduction block (Mobitz type II or $3^{\circ}$ heart blocks), patchy uptake at cardiac FDG-PET or a LGE on CMR, or gallium uptake in a pattern consistent with cardiac sarcoidosis [39].

Preliminary tests, such as ECG, chest radiography, and echocardiography, are non-specific for cardiac sarcoidosis (CS).

Abnormal electrocardiographic findings include various degrees of conduction block, such as bundle branch block (right bundle branch block more common than left bundle branch block) and fascicular block, QRS complex fragmentation, pathological Q waves, and ST-T changes. Notably, only a small proportion (3-9\%) of patient with asymptomatic cardiac sarcoidosis have an abnormal ECG [38, 39].

Echocardiographic abnormalities are variable and non-specific and are present in about $77 \%$ of patients with systemic sarcoidosis [40]. The most common features are interventricular thinning, especially basal focal areas of akinesia or dyskinesia or aneurysm, and other common findings are cardiac chambers enlargement, left and/or right ventricular systolic dysfunction, and/ or and diastolic dysfunction [40]. Granulomatous inflammation can be rarely seen as macroscopic areas of bright echoes, with a "speckled" or "snowstorm" pattern at two-dimensional echocardiography [39].

In the recent years, CMR has emerged as a valuable imaging tool for early diagnosis of cardiac sarcoidosis [41]. Thanks to its accuracy and 
resolution, it is able to detect both structural and functional abnormalities and to differentiate them from ischemic lesions. CMR has a specificity of about $78 \%$ for cardiac sarcoidosis making it the diagnostic tool of choice [41]. CMR abnormalities include not only granulomatous infiltration but also inflammation, edema, and fibrosis [41]. Different patterns of LGE can be found in patients with cardiac sarcoidosis. Early enhancement of granulomas in T2-weighted images is suggestive of the presence of inflammation and edema [41, 42]. The most common patterns of LGE distribution are subepicardial and mid-myocardial, with preferential involvement of the basal septum or inferolateral wall [43]. Right ventricular involvement has been described, too [43].

Another important imaging technique for CS diagnosis is FDG-PET. Focal or focal-on-diffuse FDG uptake, which represents active inflammation, can suggest CS. These findings have a low specificity, since these patterns are seen in other inflammatory myocardial diseases, too.

EMB is still considered an important tool for certain diagnosis of cardiac sarcoidosis. However, it has low sensitivity (25\%) due to the focal localization of lesions. Moreover, EMB is most commonly performed from the right ventricle, while disease involvement is more common in the basal septum and inferolateral LV wall, regions that are more difficult to biopsy [39]. Current consensus guidelines now suggest electrophysiological (electroanatomic mapping) or image-guided (PET or CMR) biopsy procedures to increase its sensitivity [39].

The arrhythmic risk in cardiac sarcoidosis patients raises the issue of the risk stratification of sudden death and when to consider ICD implantation. Several studies demonstrated that the only consistent association with ICD intervention in these patients was with reduced LVEF. However, a significant rate of ICD intervention occurred also in patients with low to moderate LVEF reduction, while none of those with normal LV and RV ejection fraction had appropriate ICD therapy [44]. Current consensus guidelines recommend ICD implantation in patients with known cardiac sarcoidosis and spontaneous sustained ventricular arrhythmias or prior cardiac arrest and/ or if LVEF $<35 \%$ (despite optimal medical therapy and trial of immunosuppression). ICD implantation can also be useful in patients with unexplained syncope and inducible ventricular arrhythmias. ICD implantation has also been considered at the time of pacemaker implantation (when indicated) and may be considered in patients with LVEF in the range of 36-49\%, despite optimal medical therapy for heart failure and a period of immunosuppression [39].

Interestingly, these guidelines, enhancing the role of CMR, stated that ICD implantation may be considered if patients have evidence of late gadolinium enhancement on CMR [40]. 


\subsubsection{Cardiac Catheterizations and Procedures}

\subsubsection{Coronary Angiogram}

In the diagnostic assessment of a depressed LV ejection fraction of unknown origin, coronary angiography is required to rule out coronary artery disease, particularly in patients above 35 years, males, and those carrying cardiovascular risk factors. Computed tomography angiography can be considered as an alternative, particularly if the pretest probability of ischemic disease is low to moderate.

\subsubsection{Cardiac Catheterization}

Right heart catheterization should be limited to selected cases, such as in patients with advanced disease who are candidates for cardiac transplantation, due to its limited importance in the diagnostic work-up. However it is pivotal for prognostic stratification.

\subsubsection{Endomyocardial Biopsy}

The role of endomyocardial biopsy in the diagnosis of DCM remains controversial. Modern immunohistochemical methods improve sensitivity compared with the traditional histopathological Dallas criteria [45], but endomyocardial biopsy should generally be reserved for selected cases such as patients with severe heart failure, refractory hemodynamic impairment or life-threatening arrhythmias that are potentially caused by myocarditis and might be responsive to immunosuppression or antiviral therapy [8, 46, 47]. Endomyocardial biopsy can also be useful when specific diseases with targeted treatment strategies are suspected (i.e., sarcoidosis and hemochromatosis).

\section{Box 4.3 Easily Missed: Active Myocarditis}

Myocarditis represents an underdiagnosed cause of DCM. Myocarditis is an inflammatory disease of the myocardium characterized by a great heterogeneity of presentation and evolution. Common clinical scenarios associated with myocarditis may range from subclinical asymptomatic myocarditis to perimyocarditis resembling an acute coronary syndrome, to syncope from ventricular arrhythmias or heart block, to heart failure associated with progressive or chronic DCM, to severe acute heart failure in some cases requiring intensive hemodynamic support [48].

Myocarditis can be caused by a broad range of infectious agents, including viruses, bacteria, fungi, and protozoa, as well as noninfectious triggers, such as toxins and hypersensitive reactions. Among these triggers, viral infection has been documented to constitute the most prevalent cause of myocarditis.

Clinical suspicion of inflammatory heart disease is crucial in the clinical scenarios presented above, especially in newly diagnosed DCM or in the presence of life-threatening arrhythmias: history of recent flu-like symptoms 
(present in 35-65\% of cases) or viral gastroenteritis may raise this suspicion. A previous insect bite, suspicious of Borrelia or rickettsiae, should always be investigated [8].

In patients with clinically suspected acute myocarditis, confirmatory testing usually begins with serum biomarkers. However, Troponin I may be raised in only $34 \%$ of patients with acute myocarditis [49]. Non-specific serum markers of inflammation, such as C-reactive protein, erythrocyte sedimentation rate, and leucocyte count, are frequently increased in patients with suspected myocarditis, but low specificity limits their diagnostic value.

Patients with myocarditis might mostly have non-specific changes on ECG. These include sinus tachycardia, ST wave and T wave abnormalities, and, sometimes, PR depression and diffuse ST segment elevation (if there is concomitant peri-epicardial inflammation). Electrocardiographic changes that are associated with poor prognosis in acute myocarditis include widened QRS and Q waves [50].

Echocardiography is mandatory in patients with suspected myocarditis. The entity of left ventricular dysfunction and wall motion abnormalities not associated with a coronary distribution are useful tool to suggest an acute myocarditis. In fulminant cases, there might be wall thickening due to edema [51].

Cardiac MRI sensitivity varies with clinical presentation and extent of cell necrosis [52]. In high- and intermediate-risk forms (i.e., myocarditis presenting with heart failure or arrhythmias associated with LV dysfunctions), cardiac MRI has modest diagnostic accuracy, in fact the edema may be absent in T2-weighted images since its presence highly depends by the timing of MRI performance. In patients with biopsy-proven viral myocarditis, the presence of myocardial scar, indicated by LGE, is an independent predictor of all-cause mortality and cardiac mortality, but no data are available about the prognostic value of additional cardiac MRI-related parameters, such as the pattern of distribution and the extension of LGE [53].

Endomyocardial biopsy (EMB) is the gold standard for the diagnosis of myocarditis, although its role is still controversial. Indeed, it is associated with a non-negligible rate of major complications, even in specialized centers (around 1\% of the cases) [54], and its diagnostic accuracy is still debated, highly depending on the operator experience, on the number and the location of tissue samples, and on the timing of the EMB. Therefore, EMB should be performed only in selected life-threatening scenarios, such as heart failure with severe ventricular dysfunction and/or life-threatening arrhythmias refractory to optimized medical therapy in the short term (usually 3 weeks) $[8,55]$.

Recently, practical and clinically oriented classification of myocarditis and its clinical management has been proposed based on event risk derived by clinical and laboratory presentation and short-term evolution, as seen in Fig. 4.2 [8]. 

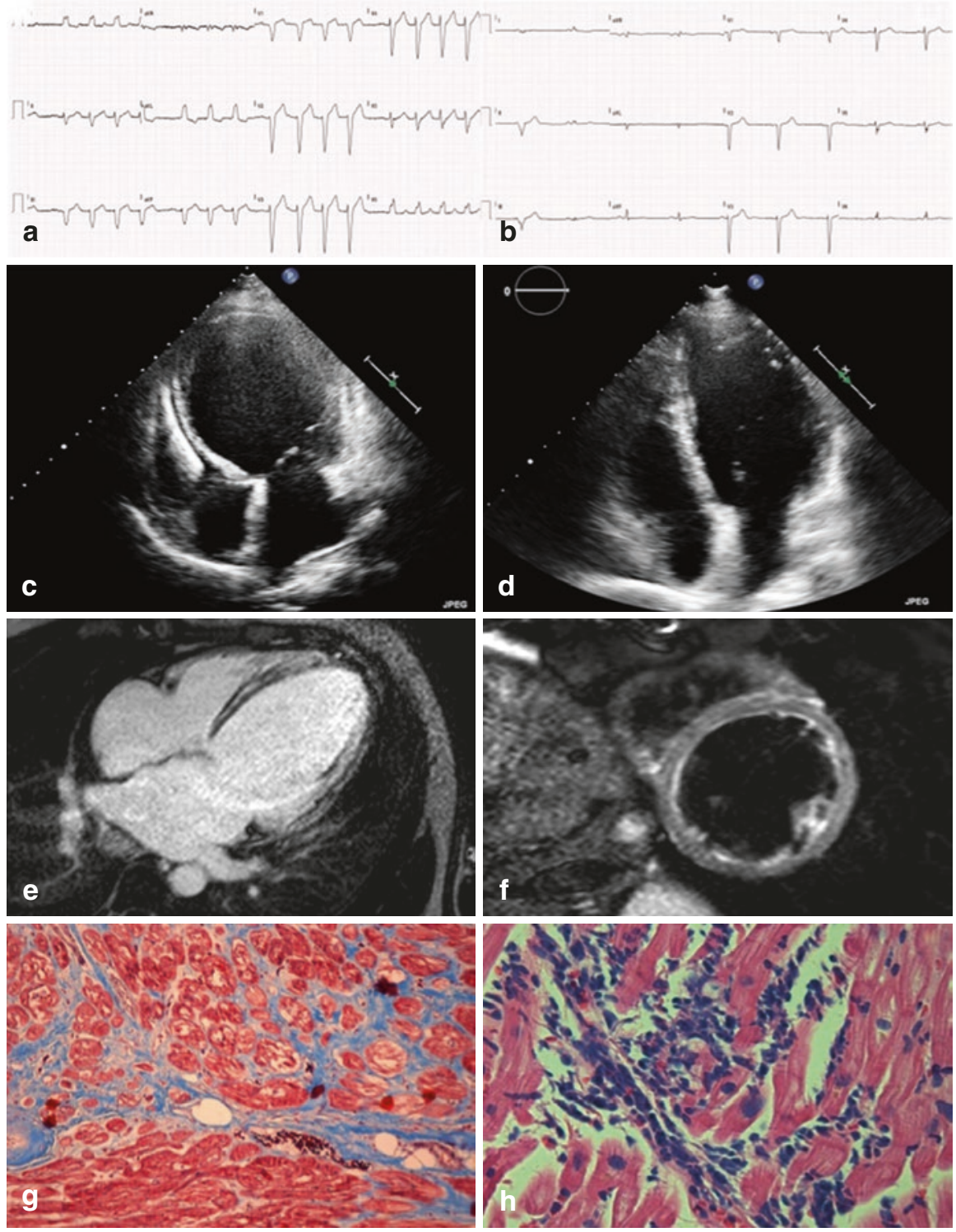

Fig. 4.2 Characterization of DCM vs. active myocarditis at diagnosis. The role of ECG (panels (a) vs. (b): note the left bundle branch block vs. low QRS voltages), echocardiography (panels (c) vs. (d): note the huge vs. mild left ventricular/atrial dilation), cardiac magnetic resonance (panels (e) vs. (f): note the mid-wall distribution pattern of late gadolinium enhancement vs. myocardial edema at T2-weighted imaging), endomyocardial biopsy (panels (g) vs. (h): note the cardiomyocyte damage and the myocardial fibrosis [in blue] vs. active lymphocytic inflammation). Reproduced with permission from Merlo et al. Evolving Concepts in Dilated Cardiomyopathy, EJHF.2018. 20(2):228-239 


\subsection{Conclusions}

New-onset dilated cardiomyopathy is still a diagnostic challenge for clinical cardiologists. It is pivotal to exclude possible removable causes of left ventricular dysfunction because this has prognostic implications. A comprehensive, systematic, and integrated approach, including third-level diagnostic tools, should be implemented in clinical practice to remove every possible reversible cause through specific therapeutic interventions. This issue appears essential to promote left ventricular reverse remodeling and subsequent outcome improvement. Excluding treatable causes is by far the most important issue. Cardiac sarcoidosis, cardiac amyloidosis, and acute myocarditis are paradigmatic examples and should be carefully excluded.

\section{References}

1. Merlo M, Pivetta A, Pinamonti B, Stolfo D, Zecchin M, Barbati G, Di Lenarda A, Sinagra G. Long-term prognostic impact of therapeutic strategies in patients with idiopathic dilated cardiomyopathy: changing mortality over the last 30 years. Eur J Heart Fail. 2014;16:317-24.

2. Merlo M, Pyxaras SA, Pinamonti B, Barbati G, Di Lenarda A, Sinagra G. Prevalence and prognostic significance of left ventricular reverse remodeling in dilated cardiomyopathy receiving tailored medical treatment. J Am Coll Cardiol. 2011;57:1468-76.

3. Rapezzi C, Arbustini E, Caforio ALP, et al. Diagnostic work-up in cardiomyopathies: bridging the gap between clinical phenotypes and final diagnosis. A position statement from the ESC Working Group on Myocardial and Pericardial Diseases. Eur Heart J. 2013;34:1448-58.

4. Merlo M, Cannatà A, Gobbo M, Stolfo D, Elliott P, Sinagra G. Evolving concepts in dilated cardiomyopathy. Eur J Heart Fail. 2018;20(2):228-39.

5. Gulati A, Jabbour A, Ismail TF, et al. Association of fibrosis with mortality and sudden cardiac death in patients with nonischemic dilated cardiomyopathy. JAMA. 2013;309:896-908.

6. Pinto YM, Elliott PM, Arbustini E, et al. Proposal for a revised definition of dilated cardiomyopathy, hypokinetic non-dilated cardiomyopathy, and its implications for clinical practice: a position statement of the ESC working group on myocardial and pericardial diseases. Eur Heart J. 2016;37:1850-8.

7. Bobbo M, Pinamonti B, Merlo M, et al. Comparison of patient characteristics and course of hypertensive hypokinetic cardiomyopathy versus idiopathic dilated cardiomyopathy. Am J Cardiol. 2017;119:483-9.

8. Sinagra G, Anzini M, Pereira LN, Bussani R, Finocchiaro G, Bartunek J, Merlo M. Myocarditis in clinical practice. A clinical approach to myocarditis. Mayo Clin Proc. 2016.

9. Mestroni L, Maisch B, McKenna WJ, Schwartz K, Charron P, Rocco C, Tesson F, Richter A, Wilke A, Komajda M. Guidelines for the study of familial dilated cardiomyopathies. Collaborative Research Group of the European Human and Capital Mobility Project on Familial Dilated Cardiomyopathy. Eur Heart J. 1999;20:93-102.

10. Moretti M, Merlo M, Barbati G, Di Lenarda A, Brun F, Pinamonti B, Gregori D, Mestroni L, Sinagra G. Prognostic impact of familial screening in dilated cardiomyopathy. Eur J Heart Fail. 2010;12:922-7.

11. Puggia I, Merlo M, Barbati G, Rowland TJ, Stolfo D, Gigli M, Ramani F, Di Lenarda A, Mestroni L, Sinagra G. Natural history of dilated cardiomyopathy in children. J Am Heart Assoc. 2016; https://doi.org/10.1161/JAHA.116.003450.

12. Anzini M, Merlo M, Sabbadini G, et al. Long-term evolution and prognostic stratification of biopsy-proven active myocarditis. Circulation. 2013;128:2384-94. 
13. Taylor MRG, Fain PR, Sinagra G, et al. Natural history of dilated cardiomyopathy due to lamin A/C gene mutations. J Am Coll Cardiol. 2003;41:771-80.

14. Sen-Chowdhry S, Syrris P, Prasad SK, Hughes SE, Merrifield R, Ward D, Pennell DJ, McKenna WJ. Left-dominant arrhythmogenic cardiomyopathy: an under-recognized clinical entity. J Am Coll Cardiol. 2008;52:2175-87.

15. Aleksova A, Carriere C, Zecchin M, Barbati G, Vitrella G, Di Lenarda A, Sinagra G. Newonset left bundle branch block independently predicts long-term mortality in patients with idiopathic dilated cardiomyopathy: data from the Trieste Heart Muscle Disease Registry. Europace. 2014;16:1450-9.

16. Ponikowski P, Voors AA, Anker SD, et al. 2016 ESC Guidelines for the diagnosis and treatment of acute and chronic heart failure. Eur Heart J. 2016;37:2129-200.

17. Hazebroek MR, Moors S, Dennert R, et al. Prognostic relevance of gene-environment interactions in patients with dilated cardiomyopathy: applying the MOGE(S) classification. J Am Coll Cardiol. 2015;66:1313-23.

18. Ortiz-Genga MF, Cuenca S, Dal Ferro M, et al. Truncating FLNC mutations are associated with high-risk dilated and arrhythmogenic cardiomyopathies. J Am Coll Cardiol. 2016;68:2440-51.

19. Herman DS, Lam L, Taylor MRG, et al. Truncations of titin causing dilated cardiomyopathy. N Engl J Med. 2012;366:619-28.

20. Haas J, Frese KS, Peil B, et al. Atlas of the clinical genetics of human dilated cardiomyopathy. Eur Heart J. 2015;36:1123-35a.

21. Lang RM, Bierig M, Devereux RB, et al. Recommendations for chamber quantification: a report from the American Society of Echocardiography's Guidelines and Standards Committee and the Chamber Quantification Writing Group, developed in conjunction with the European Association of Echocardiograph. J Am Soc Echocardiogr. 2005;18:1440-63.

22. Gigli M, Stolfo D, Merlo M, Barbati G, Ramani F, Brun F, Pinamonti B, Sinagra G. Insights into mildly dilated cardiomyopathy: temporal evolution and long-term prognosis. Eur J Heart Fail. 2017;19:531-9.

23. van Rijsingen IAW, Arbustini E, Elliott PM, et al. Risk factors for malignant ventricular arrhythmias in lamin a/c mutation carriers a European cohort study. J Am Coll Cardiol. 2012;59:493-500.

24. Pinamonti B, Zecchin M, Di Lenarda A, Gregori D, Sinagra G, Camerini F. Persistence of restrictive left ventricular filling pattern in dilated cardiomyopathy: an ominous prognostic sign. J Am Coll Cardiol. 1997;29:604-12.

25. Spezzacatene A, Sinagra G, Merlo M, et al. Arrhythmogenic phenotype in dilated cardiomyopathy: natural history and predictors of life-threatening arrhythmias. J Am Heart Assoc. 2015;4:e002149.

26. Japp AG, Gulati A, Cook SA, Cowie MR, Prasad SK. The diagnosis and evaluation of dilated cardiomyopathy. J Am Coll Cardiol. 2016;67:2996-3010.

27. Stolfo D, Merlo M, Pinamonti B, Poli S, Gigli M, Barbati G, Fabris E, Di Lenarda A, Sinagra G. Early improvement of functional mitral regurgitation in patients with idiopathic dilated cardiomyopathy. Am J Cardiol. 2015;115:1137-43.

28. Merlo M, Gobbo M, Stolfo D, et al. The prognostic impact of the evolution of RV function in idiopathic DCM. JACC Cardiovasc Imaging. 2016; https://doi.org/10.1016/j. jcmg.2016.01.027.

29. Bozkurt B, Colvin M, Cook J, et al. Current diagnostic and treatment strategies for specific dilated cardiomyopathies: a scientific statement from the American Heart Association. Circulation. 2016;134:e579-646.

30. Rapezzi C, Lorenzini M, Longhi S, Milandri A, Gagliardi C, Bartolomei I, Salvi F, Maurer MS. Cardiac amyloidosis: the great pretender. Heart Fail Rev. 2015;20:117-24.

31. Phelan D, Collier P, Thavendiranathan P, Popovic ZB, Hanna M, Plana JC, Marwick TH, Thomas JD. Relative apical sparing of longitudinal strain using two-dimensional speckletracking echocardiography is both sensitive and specific for the diagnosis of cardiac amyloidosis. Heart. 2012;98:1442-8. 
32. Hutt DF, Quigley A-M, Page J, et al. Utility and limitations of 3,3-diphosphono-1,2propanodicarboxylic acid scintigraphy in systemic amyloidosis. Eur Heart J Cardiovasc Imaging. 2014;15:1289-98.

33. Assomull RG, Prasad SK, Lyne J, Smith G, Burman ED, Khan M, Sheppard MN, PooleWilson PA, Pennell DJ. Cardiovascular magnetic resonance, fibrosis, and prognosis in dilated cardiomyopathy. J Am Coll Cardiol. 2006;48:1977-85.

34. Masci PG, Doulaptsis C, Bertella E, et al. Incremental prognostic value of myocardial fibrosis in patients with non-ischemic cardiomyopathy without congestive heart failure. Circ Heart Fail. 2014;7:448-56.

35. Satoh H, Sano M, Suwa K, Saitoh T, Nobuhara M, Saotome M, Urushida T, Katoh H, Hayashi H. Distribution of late gadolinium enhancement in various types of cardiomyopathies: Significance in differential diagnosis, clinical features and prognosis. World J Cardiol. 2014;6:585-601.

36. Puntmann VO, Carr-White G, Jabbour A, et al. T1-mapping and outcome in nonischemic cardiomyopathy: all-cause mortality and heart failure. JACC Cardiovasc Imaging. 2016;9:40-50.

37. Sekhri V, Sanal S, DeLorenzo LJ, Aronow WS, Maguire GP. Cardiac sarcoidosis: a comprehensive review. Arch Med Sci. 2011;7:546-54.

38. Mehta D, Lubitz SA, Frankel Z, Wisnivesky JP, Einstein AJ, Goldman M, Machac J, Teirstein A. Cardiac involvement in patients with sarcoidosis: diagnostic and prognostic value of outpatient testing. Chest. 2008;133:1426-35.

39. Birnie DH, Nery PB, Ha AC, Beanlands RSB. Cardiac Sarcoidosis. J Am Coll Cardiol. 2016;68:411-21.

40. Houston BA, Mukherjee M. Cardiac sarcoidosis: clinical manifestations, imaging characteristics, and therapeutic approach. Clin Med Insights Cardiol. 2014;8(Suppl 1):31-7. https://doi. org/10.4137/CMC.S15713.

41. Greulich S, Deluigi CC, Gloekler S, et al. CMR imaging predicts death and other adverse events in suspected cardiac sarcoidosis. JACC Cardiovasc Imaging. 2013;6:501-11.

42. Patel MR, Cawley PJ, Heitner JF, et al. Detection of myocardial damage in patients with sarcoidosis. Circulation. 2009;120:1969-77.

43. Hulten E, Aslam S, Osborne M, Abbasi S, Bittencourt MS, Blankstein R. Cardiac sarcoidosis-state of the art review. Cardiovasc Diagn Ther. 2016;6:50-63.

44. Betensky BP, Tschabrunn CM, Zado ES, Goldberg LR, Marchlinski FE, Garcia FC, Cooper JM. Long-term follow-up of patients with cardiac sarcoidosis and implantable cardioverterdefibrillators. Heart Rhythm. 2012;9:884-91.

45. Caforio ALP, Pankuweit S, Arbustini E, et al. Current state of knowledge on aetiology, diagnosis, management, and therapy of myocarditis: a position statement of the European Society of Cardiology Working Group on Myocardial and Pericardial Diseases. Eur Heart J. 2013;34:2636-48, 2648a-2648d

46. Cooper LT, Baughman KL, Feldman AM, et al. The role of endomyocardial biopsy in the management of cardiovascular disease: a scientific statement from the American Heart Association, the American College of Cardiology, and the European Society of Cardiology Endorsed by the Heart Failure Society of America and the Heart Failure Association of the European Society of Cardiology. Eur Heart J. 2007;28:3076-93.

47. Pollack A, Kontorovich AR, Fuster V, Dec GW. Viral myocarditis: diagnosis, treatment options, and current controversies. Nat Rev Cardiol. 2015;12:670-80.

48. Sagar S, Liu PP, Cooper LTJ. Myocarditis. Lancet (London, England). 2012;379:738-47.

49. Smith SC, Ladenson JH, Mason JW, Jaffe AS. Elevations of cardiac troponin I associated with myocarditis. Experimental and clinical correlates. Circulation. 1997;95:163-8.

50. Ukena C, Mahfoud F, Kindermann I, Kandolf R, Kindermann M, Bohm M. Prognostic electrocardiographic parameters in patients with suspected myocarditis. Eur J Heart Fail. 2011;13:398-405.

51. Mendes LA, Dec GW, Picard MH, Palacios IF, Newell J, Davidoff R. Right ventricular dysfunction: an independent predictor of adverse outcome in patients with myocarditis. Am Heart J. 1994;128:301-7. 
52. Francone M, Chimenti C, Galea N, Scopelliti F, Verardo R, Galea R, Carbone I, Catalano C, Fedele F, Frustaci A. CMR sensitivity varies with clinical presentation and extent of cell necrosis in biopsy-proven acute myocarditis. JACC Cardiovasc Imaging. 2014;7:254-63.

53. Grun S, Schumm J, Greulich S, et al. Long-term follow-up of biopsy-proven viral myocarditis: predictors of mortality and incomplete recovery. J Am Coll Cardiol. 2012;59:1604-15.

54. Chimenti C, Verardo R, Scopelliti F, Grande C, Petrosillo N, Piselli P, De Paulis R, Frustaci A. Myocardial expression of Toll-like receptor 4 predicts the response to immunosuppressive therapy in patients with virus-negative chronic inflammatory cardiomyopathy. Eur J Heart Fail. 2017;19:915-25.

55. Heymans S, Eriksson U, Lehtonen J, Cooper LTJ. The quest for new approaches in myocarditis and inflammatory cardiomyopathy. J Am Coll Cardiol. 2016;68:2348-64.

Open Access This chapter is licensed under the terms of the Creative Commons Attribution 4.0 International License (http://creativecommons.org/licenses/by/4.0/), which permits use, sharing, adaptation, distribution and reproduction in any medium or format, as long as you give appropriate credit to the original author(s) and the source, provide a link to the Creative Commons license and indicate if changes were made.

The images or other third party material in this chapter are included in the chapter's Creative Commons license, unless indicated otherwise in a credit line to the material. If material is not included in the chapter's Creative Commons license and your intended use is not permitted by statutory regulation or exceeds the permitted use, you will need to obtain permission directly from the copyright holder. 